\title{
As dificuldades do diagnóstico precoce da Síndrome de Asperger e seus impactos psicossociais na vida do paciente
}

\author{
Difficulties in the early diagnosis of Asperger Syndrome and its psychosocial impacts on \\ the patient's life
}

\author{
Las dificultades del diagnóstico precoz del síndrome de Asperger y sus impactos \\ psicosociales en la vida del paciente
}

Fernanda Vilela das Virgens ${ }^{1 *}$, Paula Mariana Gomes Castro², Lara Stefhany Oliveira Silva ${ }^{3}$, Victória Carolini Mendes ${ }^{4}$, Pedro Câmara de Assis ${ }^{1}$, Amanda dos Reis Marcelo², Gisele Vieira Meirelles ${ }^{4}$, Thalita Victória Chagas Cardoso², landra Silva Vieira1, Arthur Nolasco Gusmão Soares ${ }^{1}$.

\begin{abstract}
RESUMO
Objetivo: Analisar as dificuldades do diagnóstico precoce da Síndrome de Asperger e seus impactos psicossociais na vida do paciente. Revisão bibliográfica: A Síndrome de Asperger (AS) é incialmente manifestada na infância, especialmente entre os 5 a 9 anos de idade, entretanto, devido aos sintomas sutis e quase imperceptíveis é diagnosticada mais frequentemente na adolescência ou até mesmo na fase adulta. Sendo assim, a família e/ou cuidadores possuem uma importância significativa nesse processo de descoberta da síndrome. Estar atento ao desenvolvimento rotineiro da criança, notando atrasos na fala, ausência de contato ocular, repetição de movimentos e interesses peculiares são de fácil percepção. Procurar um especialista precocemente, oferece esse diagnóstico inicial, e consequentemente, estimula a aprendizagem da criança de forma correta. Essas intervenções podem reduzir, significativamente, as dificuldades de socialização e comportamento, assim como outras comorbidades associadas. Considerações finais: Embora seja uma patologia sutil e de diagnóstico difícil, o prognostico da SA é considerado positivo. Se realizado, precocemente, seria de extrema valia e estão associados a ganhos significativos ao paciente.
\end{abstract}

Palavras-chave: Diagnóstico precoce, Síndrome de Asperger, Impactos psicossociais.

\begin{abstract}
Objective: Objective: To analyze the difficulties of early diagnosis of Asperger Syndrome and its psychosocial impacts on the patient's life. Review bibliography: Asperger Syndrome (AS) is initially manifested in childhood, especially between 5 to 9 years of age, however, due to subtle and almost imperceptible symptoms, it is more frequently diagnosed in adolescence or even in adulthood. In addition, the family and/or caregivers play a significant role in this process of discovering the syndrome. Paying attention to the child's routine development, noticing speech delays, lack of eye contact, repetition of movements and peculiar interests are easy to perceive. Seeking an early specialist can bring this initial diagnosis, and consequently, encourage the child to learn correctly. These interventions can significantly reduce socialization and behavioral difficulties, as well as other associated comorbidities. Final considerations: Although it is a subtle pathology and difficult to diagnose, the prognosis of AS is considered positive. If performed early, it would be extremely valuable and are associated with significant gains for the patient.
\end{abstract}

Keywords: Early diagnosis, Asperger syndrome, Psychosocial impacts.

\footnotetext{
${ }^{1}$ Faculdade Santo Agostinho (FASA), Vitória da Conquista - BA. *E-mail: f_villela_v@hotmail.com

2 Centro Universitário FG (UniFG), Guanambi - BA.

${ }^{3}$ Faculdade Santo Agostinho de Itabuna (FASAI), Itabuna - BA.

4 Universidade Nove de Julho (UNINOVE), São Paulo - SP.
} 


\section{RESUMEN}

Objetivo: Analizar las dificultades del diagnóstico precoz del síndrome de Asperger y sus impactos psicosociales en la vida del paciente. Revisión bibliográfica: El síndrome de Asperger (SA) se manifiesta inicialmente en la infancia, especialmente entre los 5 y los 9 años de edad, sin embargo, debido a síntomas sutiles y casi imperceptibles, se diagnostica con mayor frecuencia en la adolescencia o incluso en la edad adulta. Además, la familia y / o los cuidadores juegan un papel importante en este proceso de descubrimiento del síndrome. Prestar atención al desarrollo rutinario del niño, notar retrasos en el habla, falta de contacto visual, repetición de movimientos e intereses peculiares son fáciles de percibir. La búsqueda de un especialista temprano puede traer este diagnóstico inicial y, en consecuencia, animar al niño a aprender correctamente. Estas intervenciones pueden reducir significativamente las dificultades de socialización y comportamiento, así como otras comorbilidades asociadas. Consideraciones finales: Aunque es una patología sutil y de difícil diagnóstico, el pronóstico de la EA se considera positivo. Si se realiza temprano, sería extremadamente valioso y se asocia con ganancias significativas para el paciente.

Palabras clave: Diagnóstico precoz, Síndrome de Asperger, Impactos psicosociales.

\section{INTRODUÇÃO}

A Síndrome de Asperger (AS) foi descrita, pela primeira vez, por Hans Asperger em 1944, mas, foi incluída no DSM-IV como critérios para diagnóstico apenas em 1994. Segundo a quarta edição do Manual Diagnóstico Estatístico de Transtornos Mentais (DSM-IV-TR), os Transtornos Invasivos do Desenvolvimento (TID) são: Transtorno Autista, Síndrome de Asperger, Transtorno Desintegrativo Infantil e Transtorno Invasivo do Desenvolvimento (COSTA A e MARIN AH, 2017).

Porém, em 2013, a Associação Americana de Psiquiatria retirou as 4 nomenclaturas anteriores e definiu o autismo em um único nome. Sendo assim, a partir do DSM-V o autismo é apenas chamando de Transtorno do Espectro do Autismo (TEA), que foi dividido em 3 graus: leve, moderado e severo. Para os diagnósticos descritos os seguintes critérios são considerados: distúrbios da comunicação social e comportamentos restritivos/repetitivos. Então, pode se afirmar, que Síndrome de Asperger é considerada um distúrbio leve (GONZÁLEZ-ALBA B, et al., 2019).

A SA é um transtorno invasivo do desenvolvimento, caracterizado por disfunção social, interesses restritos e comportamentos repetitivos. O diagnóstico da SA requer a demonstração de prejuízos qualitativos na interação social e padrões de interesses restritos, sem que exista, entretanto, um atraso clínico significativo no desenvolvimento cognitivo e da linguagem. Por outro lado, a criança apresenta como sintoma uma inteligência acima da média. Este sintoma aparece, normalmente, a partir dos 3 anos de idade com características muito sutis (MARQUES LS, et al., 2021).

Além disso, é uma síndrome, de difícil análise, dificultando muitos pais não identificarem o diagnóstico. Os sintomas podem simular outros tipos de doenças e distúrbios, contribuindo para esse diagnóstico errôneo e, consequentemente, cursar com tratamentos incorretos. Esse caráter heterogêneo ocorre, principalmente, devido a diferença de sintomatologia presentes em cada paciente; o quadro de características e hábitos, não necessariamente apresentará em todos os portadores da síndrome, sendo relativo por indivíduo (ALONSO FT, et al., 2019).

Ainda não se sabe ao certo a etiologia determinante para a síndrome, entretanto, considera-se ter uma forte contribuição hereditária, ou seja, associada ao fator genético. Ademais, alguns fatores surgiram como possíveis influenciadores para o desenvolvimento do TEA, como baixo peso ao nascer, idade dos pais, condições ambientais e prematuridade (REIS ST e LENZA N, 2020).

O portador da SA, devido à dificuldade em diagnosticar sua patologia, precocemente, apresenta desde criança/adolescente o obstáculo da construção da interação social com consequências psicossociais. Esse déficit resultará em suas vidas adultas que terão empecilhos de inserção em sociedade, seja no campo social, 
financeiro ou acadêmico, fazendo com que, muitas vezes, não consigam alcançar independência no mercado financeiro, por exemplo. Considerando o exposto, tornam-se pessoas onerosas, improdutivas e, portanto, caso as necessidades fossem atendidas poderiam ser sujeitos com SA capazes de desempenhar diversas funções (CHRYSOSTOMO HBM, 2021).

Identificar SA para a sociedade, é necessário para que realmente, realmente, seja possível agir precocemente no tratamento de crianças que apresentem sintomas. Os portadores da síndrome demonstram um espectro de sintomas mais severos que possam ser diagnosticadas, mas que, na realidade não são. Consequentemente são vistas como "diferentes" ou "anormais" ou até mesmo são diagnosticadas, erroneamente, com outros distúrbios. Incluir e reconhecer a SA, com critérios mais claros e objetivos, pode levar a uma redução de diagnósticos tardios (LOPES CN e PONCIANO VLO, 2018).

O Centro de Controle e Prevenção de Doenças (CDC), que é uma agência do Departamento de Saúde e serviços humanos dos Estados Unidos da América (EUA), tem rastreado o número e características das crianças com espectro autista. Assim, mostra-se a prevalência da doença em 2004 (de 1 a cada 166), em 2018 (de 1 a cada 59), e em 2020, a prevalência (de 1 a cada 54). Ainda, segundo a CDC, no Brasil existem em torno de 2 milhões de pacientes com TEA. Esse elevado número de diagnósticos, mostra o quanto é um problema de saúde pública. A partir disso, faz se necessário a implantação de políticas públicas e estratégias para promover o diagnóstico precoce; consequentemente, diminuir os impactos aos pacientes e familiares oferecendo qualidade de vida, com amplo atendimento adequado e especializado a essa população (SILVA EM, 2020).

Esse constructo teve como objetivo analisar as dificuldades do diagnóstico precoce da Síndrome de Asperger e seus impactos psicossociais na vida do paciente.

\section{REVISÃO BIBLIOGRÁFICA}

\section{O que é a Síndrome de Asperger}

O autismo foi classificado por Léo Karnner em 1943, como indivíduos acometidos por um distúrbio do contato afetivo, isento de linguagem e/ou expressão de linguagem, incapazes de estabelecer vínculos afetivos. Somente, em 1944, Hans Asperger, pesquisador e psiquiatra austríaco, desenvolveu um estudo com crianças portadoras de habilidades intelectuais preservados; porém, não apresentavam ou não reconheciam a comunicação não-verbal. Asperger definiu essa condição como "psicopatia autística", denominada como transtorno de personalidade, marcado pelo isolamento social (TEIXEIRA MCS e GANDA DR, 2019).

Inicialmente, o estudo de Hans Asperger não foi reconhecido internacionalmente, porém, em 1976, a psiquiatra Lorna Wing publicou um artigo, relatando a difusão da tese no mundo cientifico, e comparando ao autismo de Karnner devido similaridade entre os temas. Desde então, a "psicopatia autística infantil" passou a ser conhecida como Síndrome de Asperger (SILVA SCF e RANGNI RA, 2019).

A classificação de Transtornos Mentais e de Comportamento da CID-10 realizada pela Organização Mundial da Saúde (OMS), caracteriza a Síndrome de Asperger como um transtorno de classificação incerta. Características similares ao autismo como anormalidades qualitativas de interação social junto a um repertório de interesses e atividades restrito estereotipado e repetitivo. Destaca-se, além disso, a diferença para o autismo dar-se por não haver nenhum atraso global no desenvolvimento cognitivo ou na linguagem, embora seja comum muitos pacientes serem, marcadamente, desajeitados (FRANCA EJ, et al., 2021).

A CID-10 relata ainda ser provável alguns casos representarem algum grau de autismo leve, mas, é incerto se esse quadro estaria presente em todos os pacientes com Asperger. Há também uma forte tendência para que essas anormalidades citadas persistam desde a vida infantil até a vida adulta, sendo comum sintomas psicóticos já no início da vida (CARLOTTO MS, et al., 2019). Além disso, considera-se a Síndrome de Asperger como uma variante do Autismo; é uma forma atenuada e diferenciada, com características peculiares, além daquelas que são comuns em todas as formas de Autismo. Segundo o DSM-IV-TR, a Síndrome de Asperger é um transtorno global do desenvolvimento (PARRAGA GCV, et al., 2019). 


\section{Alterações neuroanatômicas no cérebro e aspectos biológicos}

Inicialmente, Karner em 1943, foi o primeiro a observar o aumento do tamanho da cabeça relatando "cabeças grandes" nas crianças autistas que ele examinou. Em seguida, demonstrou aumento do perimétrico cefálico fronto-occipital, por meio de medições métricas, peso cerebral e volume cerebral distintas de um desenvolvimento neurotípico (VÁSQUEZ B e SOL MD, 2017).

Atualmente, pesquisas têm apontado uma relação existente entre anormalidades cerebrais e a justificativa para SA. Com técnicas avançadas de imagem tem-se percebido diferenças estruturais e funcionais em determinadas regiões do cérebro. Há hipóteses que afirmam estar associado a alterações das densidades das substancias cinzentas ou brancas, alterações de volume cerebral e uma possível conexão atípica, que poderia dificultar o processamento de informações. Outra possibilidade biológica estaria correlacionada ao período de desenvolvimento embrionário fetal, em que as células migrariam de forma anormal durante esta fase, o que afeta os circuitos neuronais do cérebro, influenciando nos aspectos emocionais e sociais do portador de Asperger (LINDOSO AGA, et al., 2021).

Essas características biológicas e alterações neuroanatômicas são verificadas ainda durante o exame físico do paciente, em que se percebe os possíveis resultados dessas modificações. Reflexos nos pares cranianos podem ou não estar alterados, com influência no tônus, reflexos superficiais e profundos, além de possível acometimentos na coordenação motora (GONÇALVES JP, et al., 2020).

A origem dos comportamentos difíceis, apresentados por esses indivíduos pode estar relacionado ao fato de que dentro do TEA alguns autistas apresentam transtornos sensoriais, sendo eles visuais, auditivos, táteis, olfativos e gustativos, desencadeando hábitos difíceis, como ir a locais com muito barulho, restrição alimentar, comportamentos agressivos em salas de aula, sendo que até eles mesmos não sabem como lidar com esse estímulo (MOTA RCC, et al., 2021).

Há ainda uma forte correlação estudada nas últimas pesquisas feitas pelos cientistas acerca do componente genético. Tanto na SA quanto nos demais transtornos dentro do espectro autista existe uma tendência a hereditariedade, principalmente entre parentes de primeiro grau que podem ter sintomas parecidos com a SA, porém limitados, como por exemplo dificuldade de interação social leve e comportamentos restritivos. O mecanismo não foi totalmente elucidado, mas, provavelmente, exista um conjunto comum de genes, em que suas variações ou deleções possam provocar esses sintomas, que irá depender da gravidade dessas lesões genéticas em cada pessoa (VIANA ACV, et al., 2020).

\section{Diagnóstico precoce e impactos psicossociais}

A Síndrome de Asperger é incialmente manifestada na infância, especialmente entre os 5 a 9 anos de idade, entretanto, devido aos sintomas sutis e quase imperceptíveis é diagnosticada mais frequentemente na adolescência ou até mesmo na fase adulta. Além disso, tem-se as dificuldades enfrentadas pelos profissionais em reconhecer os sintomas contribuindo para esse diagnóstico tardio (VALENCIA JBZ, et al., 2018).

Os pacientes com a SA possuem algumas características que contribuem na descoberta do diagnóstico ainda nos primeiros meses de vida. Ademais, as diferenças quanto ao desenvolvimento inicial, ainda durante a amamentação, são usuais que eles desviem do contato olho a olho com a mãe, ficando mais atentos a estímulos luminosos. É possível que essa dificuldade esteja presente ao longo da vida sem o sistema motor e o sensorial caminharem juntos, além disso, costumam apresentar dificuldades de prestar atenção em todo o ambiente por possuírem somente um foco (MEJIA SDPM, et al., 2021).

Outro fator associado a esse diagnóstico tardio, diz respeito a dificuldade que é detectar a Síndrome de Asperger nos pacientes. Muitas vezes os sintomas apresentados são confundidos com outras síndromes como Transtorno de Déficit de Atenção (TDAH), Hiperatividade ou Esquizofrenia, Autismo, entre outros. Esses diagnósticos errôneos podem levar ao aparecimento de sintomas depressivos, estresse, ansiedade, transtornos compulsivos, ou seja, podem levar a problemas relacionados a regulação hormonal, aspectos físicos da saúde, alterações no sono e hábitos alimentares, afetando assim dificuldades sociais e também no mercado de trabalho (LOPES CN, 2018). 
No espaço escolar, a criança com Asperger sente-se mais isolada, preferem somente uma brincadeira ou apenas conversar sobre assuntos aos quais se interessam, embora outros indivíduos não gostem, e não raramente sofrem bullying. Entretanto, são pessoas que conseguem se adaptar as situações quando são diagnosticadas ainda nessa fase da vida e estão mediante acompanhamento médico e de outros profissionais de saúde (LIMA CM, et al., 2021).

$\mathrm{Na}$ fase adulta, por apresentarem, diferentemente das formas mais graves do autismo, a eficiência intelectual preservada e não apresentarem comportamento agressivo e problemas na fala, possuem a oportunidade de se tornarem ótimos profissionais, mediante uma rotina planejada, sem trabalhos que ofereçam riscos ou imprevistos diferentes das suas obrigações usais, pois isso os torna vulneráveis (TEIXEIRA MCS e GANDA DR, 2019).

Os adultos com SA, embora apresentem restrições nas interações sociais, podem ter uma vida social sem limitações. A SA quando comparada as outras formas do autismo, as crianças com Asperger são mais aptas a desenvolverem e serem adultos independentes em termos de família, emprego e casamento. Os estudantes que apresentam Asperger são altamente capazes de completar a faculdade e assumir a profissão escolhida, exercendo-a com competência, segundo a sua área de interesse (GABARÃO AGM, 2019).

As dificuldades de interação e expressão de sentimentos são sintomas presentes entre os pacientes e que ajudam no diagnóstico por parte dos familiares ou cuidadores. Sinais esses que se expressam em dificuldade de entender e manifestar emoções, ter um pensamento concreto e literal de determinados assuntos e não entender metáforas e ironias. A criança em desenvolvimento aprende a lidar com as emoções intuitivamente, para o Asperger, não compreender as emoções e não saber encaixa-las nos relacionamentos familiares e sociais acarreta um sofrimento e torna-o mais difícil (LYRIO ACO e AMARAL SCS, 2019).

Além disso, a família e/ou cuidadores têm uma importância significativa nesse processo de descoberta da síndrome. Estar atento ao desenvolvimento rotineiro da criança, notando atrasos na fala, ausência de contato ocular, repetição de movimentos e interesses peculiares são de fácil percepção. Procurar um especialista precocemente, pode oferecer esse diagnóstico inicial, e consequentemente, estimular a aprendizagem da criança de forma correta (FRANCISCO AB, 2020).

A investigação diagnóstica dentro do ambiente médico é clínica, por meio dos aspectos comportamentais dos pacientes e histórico familiar. Com uma anamnese detalhada, a partir de investigações sobre a gestação e condições, sendo necessária a presença tanto do portador quanto dos cuidadores vai direcionar melhor esse diagnóstico. Deve-se também investigar fatores de risco como idade dos pais, gravidez induzida ou espontânea, álcool e consumo de drogas. Podem ser utilizados exames como tomografia de crânio, análise bioquímica, eletroencefalograma além de outros exames complementares (COSTA A e MARIN HÁ, 2017).

A Síndrome de Asperger, segundo apresentado no Manual Diagnóstico e Estatístico de Desordens Mentais - DSM V, está alocada na categoria Transtornos do Espectro Autista (TEA) e traz seu diagnóstico baseado nos seguintes critérios: déficits de comunicação/interação social; presença de um padrão repetitivo e restrito de atividades, interesses e comportamentos; e presença dos sintomas desde o início da infância, que podem não se manifestar completamente até que as demandas sociais excedam o limite de suas capacidades (ALBA BG e GONZÁLEZ PC, 2020).

Todos os sintomas expostos acima de maneira isolada não são suficientes para concretização diagnóstica do quadro da SA. O diagnóstico deve ser realizado por uma equipe multidisciplinar, de forma multifatorial, considerando as peculiaridades de cada indivíduo. Obter esse diagnóstico para muitos pacientes é um alívio. Entender que existem outras pessoas na condição torna o mundo, antes desconhecido, mais fácil de ser compreendido. Esse diagnóstico precoce resulta em uma maior qualidade de vida a esses pacientes, possibilitando uma infância ou uma vida adulta saudável e integrativa para essa população (LOPES CN, 2018).

\section{Tratamento}

O tratamento do paciente Asperger pode ser inicialmente não medicamentoso. Essa abordagem deve seguir de maneira individualizada, ou seja, cada etapa de vida do paciente é considerada. No período adolescente e infantil pode-se inserir uma terapêutica mais voltada para a interação social, aspectos da 
linguagem, educação social, habilidades sociais e questões sobre sexualidade. No período adulto o foco são orientações direcionadas ao desenvolvimento na vida adulta, mercado de trabalho e moradia. Além disso, é preciso oferecer aos portadores estratégias terapêuticas que se baseiam no enfrentamento dessas dificuldades comportamentais, cognitivas e sociais (ALBUQUERQUE LVC, 2018).

Todas as áreas estão englobadas no conjunto terapêutico, ou seja, é necessário um atendimento multidisciplinar, incluindo psiquiatra ou neurologista quando necessário, psicoterapia, psicólogo, orientação familiar. Todos esses profissionais devem ser disponibilizados para que o indivíduo não agrave seus sintomas ao longo do desenvolvimento da doença e bem como procure inserir esse indivíduo na sociedade. Nesse contexto, deve-se ainda ressaltar a importância da terapia na história clínica do paciente, indagando o portador, realizando uma boa entrevista e análise mediante o apoio de estudos e teorias (SOUZA RDB e JULIANI J, 2018).

Em muitos casos o paciente Asperger vai necessitar do tratamento psicofarmacológico, em outras palavras a associação dos dois métodos tem-se mostrado muito efetiva. Essa associação pode ser iniciada quando esses sintomas trazem algum prejuízo negativo ao paciente interferindo na sua qualidade de vida. Comportamentos como impulsividade, agitação, irritabilidade e ou agressividade devem ser tratados com maior atenção. Outro ponto a ser mencionado diz respeito as comorbidades que podem aparecer e devem ser analisadas como por exemplo casos de ansiedade, depressão, TDAH, transtornos do sono e epilepsia (GABARÃO AGM, 2018).

É muito comum o paciente com Asperger apresentar sintomas de ansiedade, depressão, distúrbios do sono, sensação de tensão ou desânimo e/ou pensamentos suicidas. Nesse sentido, deve ser recomendado o tratamento de acordo as diretrizes. $O$ tratamento realizado com os inibidores seletivos da recaptação da serotonina é bem tolerado e eficaz para os transtornos relacionados à ansiedade, principalmente quando combinados a terapia. Para o tratamento da psicose, os antipsicóticos têm se mostrado eficientes, entretanto, se possível deve-se eliminar o seu uso após a remissão dos sintomas (OLIVEIRA EM, et al., 2019).

Entre os fármacos antipsicóticos mais utilizados tem-se a risperidona, que é antipsicótico atípico, olanzepina, ziprasidona, aripiprazol. A Food and Drug Administration (FDA), indica a risperidona e o aripiprazol como drogas de escolha. Todos os fármacos citados podem ocasionar efeitos colaterais como aumento de peso, síndrome metabólica, hiperprolactnemia, síndrome extrapiramidal e síndrome metabólica. Portanto, na ausência de sintomas que justifiquem seu uso, deve-se optar pelo tratamento não medicamentoso e quando necessário, o profissional especializado deve acompanhar o paciente durante todo o tratamento (COSTA GON e ABREU CRC, 2021).

Entretanto, o uso das medicações ainda é muito discutido entre os especialistas, isso porque não há um medicamento especifico para o transtorno, fazendo com que os pacientes precisem ser acompanhados de forma criteriosa pois o uso exacerbado ou contínuo de alguns medicamentos podem ocasionar efeitos indesejados. Efeitos colaterais comuns e presentes, são pacientes agressivos que automutilam ou que colocam em risco a vida de pessoas ao seu redor pelo uso irregular ou sem acompanhamento devido. Portanto, é preciso buscar alcançar formas de minimizar os sintomas visando a qualidade de vida do paciente. (REIS ST e LENZA N, 2020).

Comumente, nota-se, uma "rigidez de pensamento" no paciente portador de Asperger, ou seja, dificuldades em lidar com mudanças levando a extrema aversão a algo novo. Percebe-se, a falta de capacidade que muitos pacientes possuem em aceitar ou aderir ao tratamento, em outras palavras, o paciente Asperger possui medo, o que dificulta seu consentimento à terapêutica. Diante da complexidade do transtorno, é necessário um processo de intervenção adequada a cada indivíduo, para obtenção de resultados consideráveis do quadro clinico do portador, promovendo-os em seus desafios sociais e possibilitando-os em conquistas para sua melhor qualidade de vida (COSTA GON e ABREU CRC, 2021).

Além disso, é importante as terapias comportamentais nesses indivíduos, somado a necessidade da terapêutica correta sendo indicado o seu acompanhamento pelos profissionais de saúde. Essas intervenções podem reduzir, significativamente, as dificuldades de socialização e comportamento, assim como outras comorbidades associadas, e portanto, proporcionar um impacto positivo no bem-estar e qualidade de vida dessas pessoas e também de familiares e cuidadores (SOUZA RDB e JULIANI J, 2018). 


\section{CONSIDERAÇÕES FINAIS}

A SA trata-se de um distúrbio neurológico, com características comportamentais, sociais e emocionais comprometidas. Desde a infância, essas disfunções são responsáveis por alterar processos fundamentais como a socialização do indivíduo, trazendo assim, consequências prejudiciais para a condição de vida do portador. Embora seja uma patologia sutil e de diagnóstico difícil, o prognostico da SA é considerado positivo. Se realizado, precocemente, seria de extrema valia e estão associados a ganhos significativos ao paciente. É imprescindível que profissionais da saúde e família compreendam o paciente e assim desenvolvam em conjunto um tratamento eficaz, pois, são capazes de se desenvolver, considerando que não apresentam comprometimento grave, porém é preciso uma intervenção o quanto antes.

\section{REFERÊNCIAS}

1. ALBA BG, GONZÁLEZ PC. Pensamento visual en personas com Síndrome de Asperger. Un caso de estudio. Dedica: Revista de Educação e Humanidades, 2020; 17: 295-312.

2. ALBUQUERQUE LVC. Tratamento homeopático nas dermatoses crônicas do tipo líquen: estudo de caso revisitado após dez anos. Revista de Homeopatia, 2018; 81(3/4): 25-31.

3. ALONSO FT, et al. Juan, uma Criança com Síndrome de Asperger: Estudo de Caso de uma Boa Prática de Inclusão Educacional por meio da Aprendizagem Cooperativa. Revista Brasileira de Educação Especial, 2019; 25(1): 85-100.

4. CARLOTTO MS, et al. Prevalência de afastamentos por transtornos mentais e do comportamento relacionados ao trabalho em professores. Psicologia da UNISC, 2019; 3(1): 19-32.

5. CHRYSOSTOMO HBM. Síndrome de asperger: a singularidade da criança e a importância ativa do psicopedagogo na educação. Revista Ibero-Americana de Humanidades, Ciências e Educação, 2021; 7(2): 15-15.

6. COSTA A, MARIN AH. Processo de inclusão do adulto com Síndrome de Asperger no ensino superior. Barbarói, 2017; (49): 258-285.

7. COSTA GON, ABREU CRC. Os benefícios do uso de psicofármacos no tratamento de indivíduos com transtorno do espectro autista (TEA): revisão bibliográfica. Revista JRG de Estudos Acadêmicos, 2021; 4(8): 240-251.

8. FRANCA EJ, et al. Importância do diagnóstico precoce em crianças com Transtorno do Déficit de Atenção com Hiperatividade: revisão narrativa. Revista Eletrônica Acervo Cientifico, 2021; 35: e7818.

9. FRANCISCO AB. O potencial das pessoas com síndrome de asperger no exercício da pesquisa acadêmica e do magistério. Revista Brasileira De Educação, Cultura E Linguagem, 2020; 4(7): 87-99.

10. GABARÃO AGM. Análise das sugestões para o trabalho docente com o estudante com Transtorno do Espectro Autistasíndrome de Asperger. Revista Com Censo: Estudos Educacionais do Distrito Federal, 2018; 5(1): $237-242$.

11. GONCCALVES JP, et al. Os diferentes discursos sobre o autismo. Salão do Conhecimento, 2020; 6(6): 1-5.

12. GONŻÁLEZ-ALBA B, et al. O diagnóstico da síndrome de Asperger no DSM-5. Ajayu Órgano de Difusão Científica do Departamento de Psicologia UCBSP, 2019; 17(2): 332-353.

13. LIMA CM, et al. Promoção da saúde mental e enfrentamento ao bullying no ambiente escolar. Pesquisa, Sociedade e Desenvolvimento, 2021; 10(5): e42210514692.

14. LINDOSO AGA, et al. Aspectos clínicos da síndrome de Asperger na comunidade de uma universidade pública. Pesquisa, Sociedade e Desenvolvimento, 2021; 10(10): e55101018707.

15. LOPES CN. Autismo e família: o desenvolvimento da autonomia de um adolescente com síndrome de Asperger e a relação familiar. Revista Diálogos e Perspectivas em Educação Especial, 2018; 5(1): 53-66.

16. LOPES CN, PONCIANO VLO. Procedimentos familiares adequados para o desenvolvimento da autonomia de adolescentes com síndrome de asperger. Cadernos da Pedagogia, 2018; 11(22): 99-112.

17. LYRIO ACO, AMARAL SCS. A tecnologia como ferramenta para aprendizagem das crianças com síndrome de asperger na educação infantil. Redin-Revista Educacional Interdisciplinar, 2019; 8(1): 1-13.

18. MARQUES LS, et al. A Síndrome De Asperger: desafios para a inclusão e a importância da literatura infanto-juvenil. Revista Augustus, 2021; 26(53): 186-199.

19. MEJIA SDPM, et al. Caso de um indivíduo adulto com síndrome de Asperger não diagnosticado. MULTIMED, 2021; 25(5): 114.

20. MOTA RCC, et al. Inclusão educacional/social dos indivíduos com transtorno do espectro autista. Interfaces do Conhecimento, 2021; 3(2): 5-47.

21. OLIVEIRA EM, et al. O impacto da psicomotricidade no tratamento de crianças com transtorno do espectro autista: revisão integrativa. Revista Eletrônica Acervo Saúde, 2019; 34: e1369.

22. PARRAGA GCV, et al. Síndrome de Asperger. Recimundo, 2019; 3(4): 416-433.

23. REIS ST, LENZA N. A Importância de um diagnóstico precoce do autismo para um tratamento mais eficaz: uma revisão da literatura. Revista Atenas Higeia, 2020; 2(1): 1-7.

24. SILVA EM. Autismo: uma revisão integrativa. Revista Científica UMC, 2020; 5(3): 1-3.

25. SILVA SCF, RANGNI RA. Indicadores de altas habilidades/superdotação em aluno com síndrome de asperger: um estudo de caso. EccoS Revista Científica, 2019; (51): 1-25.

26. SOUSA DLD, et al. Análise do comportamento aplicada: a percepção de pais e profissionais acerca do tratamento em crianças com espectro autista. Contextos Clínicos, 2020; 13(1): 105-124.

27. SOUZA RDB, JULIANI J. Psicologia e autismo. Revista Terra \& Cultura: Cadernos de Ensino e Pesquisa, 2018; 29(56): 139152.

28. TEIXEIRA MCS, GANDA DR. Inclusão E Autismo: relato de caso sobre o trabalho com uma criança na educação infantil. Psicologia e Saúde em debate, 2019; 5(2): 125-135.

29. VALENCIA JBZ, et al. Teoria de la mente y empatía en niños y niñas con diagnóstico de Síndrome de Asperger. Psicogente, 2018; 21(39): 88-101.

30. VÁSQUEZ B, SOL MD. Características neuroanatômicas da Síndrome de Asperger. International journal of morphology, 2017; 35(1): 376-385.

31. VIANA ACV, et al. Autismo. Saúde Dinâmica, 2020; 2(3): 1-18. 